\title{
Translation and Localisation: A Step towards Language Digitisation
}

\author{
Dr. Khalid M Zafar ${ }^{1}$, Md. Arfeen Zeeshan ${ }^{2}$ \\ Department of Translation, SLL\&I, Maulana Azad National Urdu University, Ghachi Bowli, Hyderabad-500 0032, India
}

\begin{abstract}
Translation is the process of rendering the content and meaning of one language into other language. The Language localisation is not comparable with Translation as it involves a varied study of the target culture so that the product may be adopted under the local needs. Digital India campaign was launched by the GoI to connect rural areas with high-speed Internet networks and improving digital literacy. The phones in future will have facility for inputting text in English, Hindi and at least one more language. The third language to be supported by mobiles will be decided by the phone manufacturer or supplier. Being a cashless society, India must have digitized information of all contents. Localizing for the Indian buyer is not an easy task - especially if it is a company's first incursion into the Asian market. There are many applications and websites in Indian languages and continuously increasing. This paper attempts to cover the localized and translated applications and websites viz available in Indian languages. Further, the paper focuses on the possible ways by the GoI to recognise, support and channelize these efforts and contributions. The paper also emphasises the role of start-ups and advocates a support plan on the basis of demographic status of various languages.
\end{abstract}

Keywords: Localization, Translation, MT, Indian languages , GoI

\section{Introduction}

\section{Translation}

Translation is the process of rendering the content and meaning of one language into another language. Translation relates specially to the written words and like interpreting which is the conversion of spoken words from one language to another.

Bassnett, a scholar and professor in the Centre for Translation and Comparative Cultural Studies at Warwick University, wrote over 20 books and has great influence on the study of translation. In Bassnett's opinion, translation is not only a kind of pure lingual activity but also a kind of communication intra-culture and inter-culture. In other words, translation is not a mere linguistic transfer but a cross-cultural activity. She proposes that the cultural aspects should be taken into consideration for the study of translation. ${ }^{[1]}$ This is usually a written text documents but may include audio-visual material such as DVD, Context, Background, audience and terminology and this ensures us that translator translates the document as effective as possible.

\section{Localization}

The Language localisation is not comparable with translation activity as it involves a wide study of the target culture so that the product may perfectly be adapted under the local prerequisites. The Localisation Industry Standard Association (LISA) defines localisation as: "Localisation involves taking a product and making it linguistically and culturally appropriate to the target locale (country/region and language) where it will be used and sold." [2]

\section{Digital India}

"Digital India is a campaign launched by the Government of India to ensure that Government services are made available to citizens electronically by improving online infrastructure and by increasing Internet connectivity or by making the country digitally empowered in the field of technology. ${ }^{,[3]}$ Digital technologies have infused into various parts of our private and public life domains. Many of us depend on them in our daily life. This initiative consists of the idea to link rural areas with high-speed internet networks. "Digital India campaign was launched by the Prime Minister of India Shri. Narendra Modi on 1 July 2015 - with an objective of connecting rural areas with high-speed Internet networks and improving digital literacy. The vision of Digital India programme is inclusive growth in areas of electronic services, products, manufacturing and job opportunities etc. and it is centred on three key areas Digital Infrastructure as a Utility to Every Citizen, Governance \& Services on Demand and Digital Empowerment of Citizens." [4]

Native language is the device of credentials in this world. This gives us a medium to preserve our own rich cultural heritage, traditional values, and all the valuable knowledge for generation yet to born. The history, passion, and soul of our own culture lives in each word we speak in our native tongue. Language is more than a system to communicate with others. According to former President of South Africa and Noble laurate Nelson Mandela "If you talk to a man in a language he understands, it goes to his head. If you talk to him in his language, it goes to his heart." ${ }^{[5]}$

There is an inevitable need for the business companies and service providers to know more about how to localize smartly and break the language barriers between them and their customers, Brian Coyle's webinar titled, 'Cross Border Selling: Breaking the Language Barrier with Automated Translation,' discussed how automated translation can help overcome the eCommerce language barrier to successfully sell across multilingual borders. Both the translation technology and language localisation can play a vital role in achieving the Digital India dream. 


\section{International Journal of Science and Research (IJSR) \\ ISSN (Online): 2319-7064}

Index Copernicus Value (2016): 79.57 | Impact Factor (2015): 6.391

\section{Localisation in Indian languages}

India is a multi-lingual country where language varies region to region and occupation to occupation etc. According to research firms there are 350 million smartphone users in the country, of which an odd 100-120 million users are fluent with English. The others would prefer their vernacular language over English as a preferred mode of communication. This left us with a huge market size to foray into. Further, reports also suggest that by 2018 , India will have close to 850 million smartphone users, which will give birth to an even larger vernacular market. India's internet users have more faith in content that's not in English. A recent study of 4,612 urban citizens and 2,448 rural Indians by management consultancy KPMG India and search giant Google found that nearly $70 \%$ of Indians consider local language digital content more reliable than English content. Of all the internet-using native speakers of an Indian language, most prefer Hindi, the co-official language of the Indian union along with English. By 2021, an expected 201 million Hindi users $-38 \%$ of the Indian internet user basewill be online, according to the KPMG-Google study. ${ }^{[6]}$

\section{Indian languages are vital}

There is a big question that why there is a need of translation of English content into Indian languages? Let's discuss it in a bit detail:

As it is mentioned, Indian language interfaces are necessary for those users who aren't particularly familiar with English, and intuitively, it will help new users become comfortable with transacting. Businesses wouldn't want Internet usage to be limited to the users who are familiar with English: that's a fraction of the country's population.

According to a government notification accessed by The Sunday Standard, the phones will have facility for inputting text in English, Hindi and at least one additional Indian language. The third language to be supported by mobiles will be decided by the phone manufacturer or supplier based on market demand. The note states, "Message readability will be provided for all 22 Indian official languages and script supporting these languages." The government is expected to bring a notification of mandatory standards soon and all mobile devices sold after December 2016 will have multiple language facility. ${ }^{[7]}$

During demonetization, Undoubtedly, we can see Cashless demand has increased. "The Indian payment system is rapidly transiting to more and more IT based systems. In the retail sector we have very high volumes of money transactions. Other than cash, one of the growing payment methods adopted by merchants in the sector is payment cards." ${ }^{[8]}$ Being a cashless society, we must have digitized information of all contents. Paytm, mobikwik etc companies added content in regional languages and emerged to be the most acceptable applications. To make it a huge success and spread awareness, some translation companies like ebhasha setu contributing effort for digital India programme in collaboration with C-DAC, Hyderabad, IIIT Hyderabad etc. During discussion of translation and digital India programme we can't overlooked the efforts of C-DAC.
Public and Private Sector organization goal is to make payments and commerce more inclusive, and this new feature will help us expand the market to include those users who would prefer to engage on line in their native languages.

Marketers know the demand of Consumers and this time they recognize the problem of Indian society. What is the main barrier for conveying message. Obviously Indian population resides in villages where regional languages being preferred/used. After many research, finally they found there is a language barrier, there is a communication gap and now translation industry going with all path to remove barrier and making it easier and.

We are living in the era of Globalization and smart phone consumers are increasing very rapidly. To make life more easier and smarter we are using best application and different web sites. Present scenario of Indian market where govt is promoting for digital payments or cashless India.

Localizing for the Indian buyer is not an easy task especially if it is a company's first incursion into the Asian market. The sensitivity, beliefs and buying behaviours of the Indian customer is as diversified and assorted as the population of the country. Therefore, localizing in India needs added planning, approach and cultural consideration. The chief aspect to keep in observance while planning to localize in India is the sheer variety of languages spoken in India. Indian Govt. policies and considerable support to the companies especially the start-ups will definitely pave the way towards digital India. Today there are quite a good number of applications and websites are available in Indian regional languages and this number is continuously increasing day by day. Here a few of them are included to show the glimpse of the scenario.

Paytm- Alibaba-backed Paytm Mall has introduced 10 Indian languages which includes Hindi, Tamil, Telugu, Gujarati, Marathi, Bengali, Kannada, Malayalam, Oriya and Punjabi. ${ }^{[9]}$

Quikr- India's No, 1 cross category classifieds business, launched vernacular language support for consumers accessing Quikr's platform. Quikr allows consumers to choose from seven different languages- Hindi, Tamil, Telugu, Kannada, Malayalam, Gujarati and Marathi to browse as well as post ads in. ${ }^{[10]}$

Gaana's Android app- Gaana has released a new update to its Android app to add support for 9 Indian languages including Hindi, Tamil, Telegu, Kannada, Malayalam, Marathi, Bengali, Punjabi, and Bhojpuri ${ }^{[11]}$

Book my show- India's largest online entertainment ticketing brand, has now added four more regional languages to its multilingual interface. Users now also have the option to conveniently browse and transact on BookMyShow website and Android app in Marathi, Malayalam, Gujarati and Punjabi in addition to the earlier available Hindi, Tamil, Telugu, Kannada and the default English option. ${ }^{[12]}$

\section{Volume 6 Issue 12, December 2017}




\section{International Journal of Science and Research (IJSR) \\ ISSN (Online): 2319-7064}

Index Copernicus Value (2016): 79.57 | Impact Factor (2015): 6.391

eBhasha Setu- It is a Language Technology start-up based out of Hyderabad. It provide Translation as a service for all the major Indian languages, Hindi, Urdu, Punjabi, Telugu and Tamil. ${ }^{13]}$

HPGas- HPCL is a Government of India Enterprise with a Navratna Status, and a Forbes 2000 and Global Fortune 500 company. It had originally been incorporated as a company under the Indian Companies Act 1913, available in 12indian languages including Malayalam, Marathi, Urdu, Hindi, Bengali, Assamese, Punjabi, Kannada, Tamil and Telugu. [14]

Digital India-11 languages-Assamese, Bengali, Gujarati, Kannada, Malayalam, Marathi, Oriya, Punjabi, Tamil, Telugu. ${ }^{[15]}$

PMO- The official website of Prime Minister's Office is now available in six major regional languages-including Gujrati, Marathi, Malayalam, Tamil, Telugu, and Bengalias part efforts to reach out to people across the country. ${ }^{[16]}$

Your Story- YourStory.com is India's no.1 media platform for entrepreneurs, dedicated to passionately championing and promoting the entrepreneurial ecosystem in India, available in 13 Indian languages including Malayalam, Marathi, Urdu, Hindi, Bengali, Assamese, Punjabi, Kannada, Tamil and Telugu. ${ }^{[17]}$

Opera Mini-Opera Mini v14.0.2 for Android adds support for more than 90 languages, including 13 Indian languages Assamese, Bengali, Gujarati, Hindi, Kannada, Kashmiri, Malayalam, Marathi, Oriya, Punjabi, Tamil, Telugu, and Urdu. ${ }^{[18]}$

Daily hunt- It is the first multilingual app on the Windows 10 platform, that provides content in 14 Indian languages just got upgraded to a whole new level of awesome! [19]

State bank buddy- "The Wallet which has been launched in English, Hindi and 11 other languages has several features like send money to registered and new users, ask money and send reminders to settle dues, transfer additional cash into an account of your choice free of cost, recharge and pay utility bills instantly, book for movie tickets, flights and hotel and shop for merchandise," [20]

Data-mail- "The linguistic email service offered by BSNL will have DataOne.Bharat domain and email address will be offered in Hindi, Gujarati, Urdu, Punjabi, Tamil, Telugu, Bengali and Marathi." i.e for 8 Indian Languages: ${ }^{[21]}$

Vikaspedia- Vikaspedia is part of democratisation of information. The portal at present can be accessed in five Indian languages. In near future it will be accessible in all 22 Indian languages. ${ }^{[22]}$

Mobikwik- Mobikwik had added Telugu and Gujarati to its list of language options, apart from English and Hindi. Its latest version has 8 languages: English, Bengali, Hindi, Gujarati, Punjabi, Odia, Tamil and Telugu. ${ }^{[23]}$
Adoption of Indic languages has increased as well - MHrd Govt.of India, BookMyShow, Amazon's Kindle, Quikr, Snap deal etc., all have at least Hindi interfaces, and most support additional languages like Tamil, Telugu etc. Swift Key and Facebook recently rolled out Hindi transliteration tools, while Google started displaying various search information in Hindi. Importantly, in December last year, BIS mandated Indian languages for handsets, which could encourage features like detecting the default language on the phone and applying it to websites etc.

\section{Machine Translation (MT) Public and Private Sector}

Machine Translation (MT) is a sub-field of Artificial Intelligence (AI), which translates the text from one language known as source language into the text of another language known as target language. Machine translation now became the most encouraging field for the researchers in India. Lots of research projects are going on machine translation for the Indian languages and between English to Indian translation and vice versa.

\section{Public Sector}

The initial work on Indian Machine Translation (in the beginning of 90's) was performed at various locations by different persons like IIT Kanpur, Computer and Information Science department of Hyderabad, NCST Mumbai, CDAC Pune, department of IT, Ministry of Communication and IT Government of India. In the mid 90's and late 90's some more machine translation projects also started at IIT Bombay, IIT Hyderabad, Department of computer science and Engineering Jadavpur University, Kolkata, JNU New Delhi etc. Some educational institutes with IIIT Hyderabad, IIT Bombay, IIT Madras etc. created language labs where they devise products and conduct research programmes. ${ }^{[24]}$ MANUU has also developed a state of the art Translation lab to carry out research work in the field of Urdu Vs Indian languages Machine Translation. MANUU is the member institution in the DietY funded and IIIT, Hyderabad lead ILMT consortium in its Phase-II which developed Sampark (MT System for Indian Languages). In fact, C-DAC is continuously contributing to the dream of digital India by doing all possible translation in regional languages and it already offers free down loadable keyboards and fonts for Indian languages.

\section{Private Sector}

Google Translate- Google is also trying to improve his MT system. Google team is very much focused on translating text into regional languages. In addition to this google keyboard available in some regional languages. "Google CEO Sundar Pichai anticipated that his company's real time automatic translation feature to be widely available in less than five years. This will break down language barriers in communication all over the world and will be especially useful in a multi-lingual country like India." [25]

ebhashasetu- ebhashasetu also developed a MT system for two pair of languages i.e.Hindi-Urdu \& Hindi-Punjabi. 


\section{International Journal of Science and Research (IJSR) ISSN (Online): 2319-7064 \\ Index Copernicus Value (2016): 79.57 | Impact Factor (2015): 6.391}

ebhashasetu is a start-up based company in IIIT Hyderabad.

Bing Translation- The Bing is an online multilingual MT platform. It was launched by the Microsoft Corporation as part of its Bing services to translate the Source Language (SL) text into the Target Language (TL) text. Till Nov 2014, the translator provided translation services in forty-five languages from around the world. The Bing is a statisticalbased platform. ${ }^{[27]}$

\section{Conclusion}

Firstly, it is the need of the hour to recognise, support and channelize these efforts and contributions. Also, inclusion of more Indian languages and more focusing on various research activities in the field of (Natural Language Processing) NLP is inevitable. Secondly, the technologies developed should made user friendly and available to all users. A robust awareness drive be launched to improve the usability of the technological resources. This could be done by a comprehensive and long-term vison based programme by the Govt. of India in a wider scope including the private sector also significantly. The role of start-ups like ebhashasetu should get the proper appreciation and support. The support plan could be prioritised on the basis of demographic status of various Indian languages, but languages with moderate and marginal demography should also be considered accordingly. Serious effort in both the areas of Translation technology and Localisation will lead country to become Digital India.

\section{References}

[1] LONG Jixing, "Translation Definitions in Different Paradigms" Canadian Social Science Vol. 9, No. 4, 2013, pp. $107-115$

[2] Tuomo Hippala "The localisation of Advertising Print Media as a Multimedia process" published on page 97 in "Multimodal Texts from Around the World: Cultural and Linguistic Insights" edited by Wendy L. Bowcher, 2012 Palgrave Macmillan, UK

[3] Shaik Shafiullah \& TVV Gopala Krishna, "Digital India: The Future of India", International Research Journal of Computer Science (IRJCS), Issue 12, Volume 3, Dec 2016

[4] Ibid

[5] http://www.bbc.co.uk/worldservice/learningenglish/movin gwords/shortlist/mandela.shtml

[6] https://qz.com/972844/indias-internet-users-have-morefaith-in-content-thats-not-in-english-study-says/

[7] http://www.livemint.com/Industry/tAMIQv9Etdeg17HirI0 $\mathrm{n} 8 \mathrm{H} /$ Indian-languages-support-in-mobiles-to-be-mademandatory.html

[8] Pranjali A. Shendge, Bhushan G. Shelar, Smitaraja S. Kapase, "Impact and Importance of Cashless Transaction in India", International Journal of Current Trends in Engineering \& Research (IJCTER), Volume 3 Issue 4, April 2017 pp. 22 - 28, http://www.ijcter.com

[9] Varsha Bansal, "Paytm Mall now supports 10 regional $\begin{array}{llll}\text { languages, } & \text { Oct } & 02, & \text { 2017, }\end{array}$ https://economictimes.indiatimes.com/smallbiz/startups/paytm-mall-now-supports-10-regionallanguages/articleshow/60905688.cms
[10] http://www.dqindia.com/quikr-goes-vernacular-breaks-thelanguage-barrier/

[11] https://tech.economictimes.indiatimes.com/news/mobile/ga anas-android-app-can-now-be-used-in-9-indian-languagesios-coming-soon/55876638

[12] http://www.freepressjournal.in/business/bookmyshowexpands-its-multilingual-interface/ 1152724

[13] www.ebhashasetu.com/html/about.html

[14] http://www.hindustanpetroleum.com/taxonomy/term/16

[15] http://meity.gov.in/sites/upload_files/dit/files/Digital\%20In dia.pdf

[16] http://www.thehindu.com/news/national/pmo-websitelaunched-in-six-regional-languages/article8663202.ece

[17] Rakesh Sidana, I want to Fly, where are my Wings: The motivational book for students, startups and entrepreneurs, Jan 2015, Parfact Printer, Gurgaon, Haryana, India. (https://books.google.co.in/books?isbn=1506019439)

[18] https://gadgets.ndtv.com/apps/news/opera-mini-for-addsbrings-support-for-13-indian-languages-improveddownload-manager-795158

[19] https://blog.dailyhunt.in

[20] Beena Parmar Mumbai, Aug 18 http://www.thehindubusinessline.com/money-andbanking/sbi-launches-mobile-wallet-in-13languages/article7554094.ece

[21] http://www.thehindubusinessline.com/info-tech/bsnldatamail-tie-up-for-free-email-addresses-in-8-regionallanguages/article9438713.ece

[22] https://www.ndtv.com/india-news/government-launchesvikaspedia-website-for-local-content- development-tools551222

[23] Nikhil Pahwa, https://www.medianama.com/2017/01/223mobikwik-lite-adds-telugu-gujarati/

[24] http://www.ijcst.com/vol31/2/sitendra.pdf

[25] Kim Arora and Vikas Singh, https://timesofindia.indiatimes.com/business/indiabusiness/if-you-dont-fail-sometimes-you-are-not-beingambitious-enough-pichai/articleshow $/ 56345353 . \mathrm{cms}$

[26] www.ebhashasetu.com/

[27] https://www.bing.com/translator

\section{Author Profile}

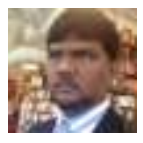

Dr. Khalid M Zafar, is Associate Professor and Head Department of Translation at Maulana Azad National Urdu University. He did his PhD in Physics and MA in Urdu literature. Since 2005 he is involved in teaching and research in the field of Translation Studies at MANUU. His core areas of research are Translation studies, Machine Translation and Scientific and Technical Translations. He is associated with the DietY funded consortium of Indian Language to Indian Language Machine Translation (ILMT) which developed Sampark and headed by IIIT, Hyderabad. 4 scholars got awarded Mphil in Translation studies under his guidance and 2 more are pursuing along with 5 pursuing $\mathrm{PhD}$. He has sound knowledge of Urdu along with the exposure to computational linguistics and Machine Translation.

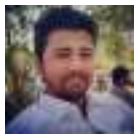

Md. Arfeen Zeeshan is pursuing M.Phil in Translation Studies from MANUU. He has very good exposure in computational linguistics and Machine Translation. He is practically working on Sampark pipeline to produce Urdu Translations from Indian language sources. 